\title{
Fragmentalist Presentist Perdurantism
}

\author{
Samuele Iaquinto* \\ To appear in Philosophia
}

Abstract: Perdurantists think of continuants as mereological sums of stages (that is, sums of instantaneous spatiotemporal parts) from different times. This view of persistence would force us to drop the idea that there is genuine change in the world. By exploiting a presentist metaphysics, Brogaard (2000) proposed a theory, called presentist four-dimensionalism, that aims to reconcile perdurantism with the idea that things undergo real change. However, her proposal commits us to reject the idea that stages must exist in their entirety. Giving up the tenet that all the stages are equally real could be a price that perdurantists are unwilling to pay. I argue that Kit Fine (2005)'s fragmentalism provides us with the tools to combine a presentist metaphysics with a perdurantist theory of persistence without giving up the idea that reality is constituted by more than purely present stages.

Keywords: Tense realism - Presentism - Fragmentalism - Perdurantism - Stage View

\section{Presentism and Perdurantism}

Perdurantists identify concrete particulars, such as tables, cats, and persons, with mereological sums of stages from different times. Stages are taken to be instantaneous spatiotemporal parts. These sums would perdure, that is, they would persist by having stages belonging to different times (cf. Lewis 1986). According to many

\footnotetext{
*Centre for Philosophy of Time, University of Milan.
} 
philosophers, there are strong reasons to prefer perdurantism over other theories of persistence (see Sider 20oI: 74-209 for an overview). To mention just one of these reasons, in contrast to endurantism (the view that concrete particulars persist by being "wholly present" at every instant at which they exist), perdurantism provides elegant solutions to the well-know puzzles involving coinciding objects』

Despite its undeniable virtues, perdurantism forces us to abandon our common-sense belief that there is genuine change in the world (Lombard 1986, Sider 200I: 212-216). Consider Alan, who is now sitting and then standing. In a perdurantist framework, this putative case of change should be described in terms of mere qualitative variations of Alan's temporal parts. The mereological sum called 'Alan' has a stage having the property of being sitting. At a later point of the sequence of Alan's stages we find another stage having the property of being standing. Despite the apparences, no entity gains or loses properties. In a sense, perdurantism invites us to adopt an atemporal perspective. From that perspective, reality appears to be nothing but a static whole.

Yet not everyone agrees that perdurantism is irreducibly incompatible with the idea that there is genuine change. In particular, Brogaard (200o) offered a temporal ontology - called presentist four-dimensionalism according to which concrete particulars have

four dimensions in the sense that they have an unfolding temporal dimension in addition to the three spatial ones [...] No stage is wholly present at more than one time; every stage is wholly present at exactly one time. There is a new stage for every moment at which a given thing exists (Brogaard 2000: 343$)$.

In describing reality as a relentless process of creation and annihilation of purely present stages, presentism would allow us to maintain that there is genuine change without loosing all the aforementioned advantages of perdurantism.

Still, there is a crucial (and quite obvious) objection against Brogaard's view: perdurantism is usually regarded as incompatible with presentism (Heller 1992). Borrowing Fine (2005: 299)'s terminology, let us treat presentism as the conjunction of the following two tenets.

\footnotetext{
${ }^{\mathrm{I}}$ See Sider (200I: 140-208) for more details.
} 
The straightforward reason why the conjunction of Ontic Presentism and Factive Presentism is seen as incompatible with perdurantism is the following. If there were no time except the present, then nothing could have more than one stage, the present one. But then nothing could be a sum of stages from different times!

In preventing this objection, Brogaard insists that

[it] rests on the idea that objects must have their temporal parts in the same way that they have their spatial parts. That is, temporal parts, like spatial parts, must exist in their entirety. This does indeed hold of those smallest temporal parts which are our successive stages. But it does not hold of temporal parts in general. That this need not be a problem is seen in the fact that events are commonly understood as having temporally extended parts even though these never exist as a whole but only through their successive stages. Similarly, objects, such as you and me, may have extended temporal parts even though these are parts which exist always only in the sense that they unfold themselves, incrementally, through their successive stages (Brogaard 2000: 346. Italics mine).

I don't find this reply convincing. In particular, I share the concerns expressed by Benovsky (2009: 297): it remains hard to see how to combine the perdurantist assumption that concrete particulars are temporally extended entities - in the sense that every stage of which they are composed is part of reality - with the claim that only one stage at a time is properly real. Giving up the idea that all the stages are equally real could be a price that perdurantists are unwilling to pay. My aim in this paper is to show that fragmentalism, a non-standard version of tense realism proposed by Kit Fine (2005): $]^{2}$ can be fruitfully employed to combine a presentist metaphysics with perdurantism. As I will argue, within a fragmentalist framework there is no need to reject the tenet that temporal parts, like spatial parts, must exist in their entirety. Put differently, if I am right in insisting that fragmentalism provides us with the tools to reconcile a version of presentism with a perdurantist theory of persistence, my view

\footnotetext{
${ }^{2}$ See also Fine (2006).
} 
will be finally able to reconcile the idea that things undergo real change without dropping the view that reality is constituted by more than purely present stages.

\section{Fragmentalist Presentism}

Standard tense realism (hereinafter, StTR) is the view that irreducibly tensed facts, such as the fact that Alan is running, obtain within a coherent reality in an absolute manner. In Fine (2005: 270-2)' 3 terminology, StTR can be thought of as the conjunction of the following three claims:
Realism Reality is constituted (at least, in part) by tensed facts.
Absolutism The constitution of reality is an absolute matter, i.e. not relative to a time or other form of temporal standpoint.
Coherence Reality is not contradictory; it is not constituted by facts with incompatible content.

Now, StTR proves to be incompatible with the following thesis (cf. Fine 2005: 27I):

Neutrality With respect to what facts constitute reality, no time is privileged.

Whoever conjoins the former and the latter - Fine (2005: 272) $4^{4}$ holds - is forced to conclude that incompatible tensed facts obtain together in an absolute sense, so ending up with a version of McTaggart (1908)'s Paradox 5 Consider the case in which Alan is running at a given instant $t_{0}$ and then sitting at $t_{1}$. Under the hypothesis that Realism holds, at $t_{0}$ reality will be constituted by the tensed fact that Alan is running, while at $t_{1}$ it will be constituted by the tensed fact that Alan is sitting. In light of Neutrality and Absolutism, even though the two facts obtain at different times, they are metaphysically on a par and constitute reality absolutely speaking. But this picture contradicts Coherence, by describing reality as containing incompatible facts.

In order to escape McTaggart's Paradox, we can go for a non-standard version of tense realism. Fragmentalism is the non-standard view that upholds both Absolutism and Neutrality while rejecting Coherence. For the

${ }^{3}$ Cf. Fine (2006: 399-400).

4 See Fine (2006: 400).

5 Obviously, the whole argument is based on the assumption that reality is complex enough to allow for qualitative variations through time. 
fragmentalist, the temporal dimension is divided up into maximally coherent collections of tensed facts, which are called fragments (Fine 2005: 28I). In what follows, I will take fragmentalism to be a pluralist view of reality, namely the view according to which there is a sharp distinction between constitution and obtainment. While constitution should be understood as absolute (bear in mind Absolutism), obtainment is irreducibly limited to a given fragment. I am aware that resorting to a version of ontological pluralism is not the only way to interpret Fine's view 6 The reason why I opt for this interpretation is that I consider it closer to the spirit of fragmentalism. Note that one of the core idea of Fine's view is that "in stating that a fact belongs to reality, we adopt a general perspective, but in stating that a fact obtains, we adopt the current perspective" (Fine 2005: 297. Italics mine). Here I will take these perspectives to mark two metaphysically fundamental features of reality. When it comes to tell what tensed facts constitute reality in an absolute manner, our fragmentalist will adopt the general perspective. In describing a fact as obtaining, instead, she will adopt the current perspective. Reconsider Alan, who is now running and then sitting. Looking from the general perspective, that is, looking at the temporal dimension as a whole, reality will be constituted - in an absolute manner - by a fragment $F_{0}$ containing the fact that Alan is running and by another fragment, $F_{1}$, containing the fact that Alan is sitting. From the current perspective, that is, from the perspective centred on the fragment we live in - say, $F_{0}$ - there is only one fact properly obtaining: the fact that Alan is running. This means that looking from the inside of $F_{0}$, there is no trans-temporal fact "out there" about Alan's being presently sitting. Analogously, when the perspective is centred on $F_{1}$, we cannot find any trans-temporal fact about Alan's being presently running. It follows that there can be no "über-fragment” in which the fact that Alan is running and Alan is sitting obtain together. Thus, even though the whole of reality is constituted by incompatible facts, the fragmentalist is not bound to admit true contradictions: the obtainment of tensed facts is always limited to a given fragment, and by hypothesis no fragment is internally incoherent (cf. Fine 2005: 282).

In order to explore a non-standard presentist metaphysics, I will reinterpret Ontic Presentism and Factive Presentism, respectively, as the theses that

\footnotetext{
${ }^{6}$ Martin Lipman (2015), for instance, examines a primitive notion of coherence in terms of co-obtainment of tensed facts.
} 
Fragmentalist $O P \quad$ Within each fragment, only present entities exist.

Fragmentalist FP Within each fragment, only presently obtaining facts obtain.

Let us call the conjunction of Fragmentalist OP and Fragmentalist FP Fragmentalist Presentism. Notice that, from the general perspective, all the fragments are metaphysically on a par. The reasons why I take my view to be a form of presentism, despite the fact that, from the general perspective, I allow for the existence of more than one fragment at a time, are that (i) the ontology of every fragment is a presentist one and (ii) obtainment is always limited to a given fragment. In other words, I assume that (i) there is no fragment containing past or future entities and (ii) from the current perspective, there is no trans-temporal fact about the existence of past or future entities located in other fragments. The fragment we live in contains neither Caesar nor Martian outposts. And from the current perspective we are by no means able to find facts about their existence. In the spirit of presentism, from the current perspective they simply do not exist. It might be replied that such a picture is somehow incoherent: the very same object - say, Caesar - exists and doesn't exist depending on the perspective we decide to adopt. But notice that this is precisely what we should expect from a version of tense realism which drops Coherence in favour of Absolutism and Neutrality. It might still be replied that this exotic version of "presentism" is not presentist at all. After all, the idea at the root of presentism is that, absolutely speaking, only present things exist. But in light of the view just described, the general perspective contains objects located in more than only one fragment. The general perspective recalls the eternalist ontology invoked by moving spotlighters. I completely agree: my view is blatantly incompatible with presentism, in the sense that it is blatantly incompatible with standard presentism. The notion of obtainment I'm employing here is deeply different from the one generally adopted by standard presentists. But that's the point. Since obtainment is thought of as limited to the fragment we inhabit, the ontology which my tense realist is committed to can contain no facts about the existence of objects located outside the present. And this is enough, I think, to vindicate the core idea of presentism: look outside the present and you won't see anything. I call this version of presentism, the one in which we adopt the non-standard notion of obtainment just described, non-standard presentism. Fragmentalist 
Presentism is a presentist view as far as non-standard presentism is concerned. Do you still feel uncomfortable with the term "presentism" when applied to my view? Just change the label. Here is another one: Fragmentalist Moving Spotlight. The crucial point here is just that as far as (i) the inventory of each fragment do not contain past or future entities and (ii) the notion of obtainment is taken to be non-standard, we have all the tools to vindicate the aforementioned intuition: from the current perspective, past and future are simply unreal.

\section{Sequence of Fragments}

Let us see how to reconcile Fragmentalist Presentism with the tenet that concrete particulars perdure. My idea is simple. I will start by assuming that fragments contain the stages that perdurantists usually describe as spread throughout spacetime regions. To this end, I will reinterpret Fragmentalist OP as the thesis that:

Fragmentalist $O P^{*} \quad$ Within each fragment, only present stages exist.

The next step is to put the fragments in a sequence able to play the role of a temporal succession of instants ordered by the earlier-than relation. Before discussing this point, let me say something more about the pluralist view I'm exploring here. As I said, reality can be described in two metaphysically fundamental ways. On the one hand, we can resort to the general perspective in order to talk about constitution in an absolute manner. On the other hand, we can switch to the current perspective and talk about tensed facts as presently obtaining in the sense described above. It should be clear that the fragmentalist cannot adopt at the same time both the general perspective and the current perspective. From the general perspective the inventory of the world is far more rich, since it includes all the tensed facts constituting reality, which are more than those obtaining, from the current perspective, in just one fragment at a time. Still, what happens in the general perspective is not completely independent of what happens in the other one, and vice versa. In particular, it seems intuitive to say that, if a fact obtains in at least one fragment, then ipso facto it constitutes reality. And it would be surprising to find out facts constituting reality from the general perspective that do not obtain somewhere in at least one fragment. If a fact constitutes reality, then there must be at least one fragment in which that very fact obtains. Even though 
the inventory of the world changes as we change perspective, facts obtaining in each fragment are the same facts constituting reality from the general perspective. And facts constituting reality from the general perspective are somehow bound to obtain in at least one fragment. Now, since from the current perspective we can find no trans-temporal fact connecting fragments, it seems reasonable to conclude that no trans-temporal fact can constitute reality from the general perspective. This is the reason why, even looking from the general perspective, reality should be described as fragmented: reality is not "of a whole" (cf. Fine 2005: 262). Let me now forestall a possible reply. From the current perspective there are no facts able to "connect" fragments, since facts obtain only within them, and there is no "über-fragment" encompassing them all within which trans-temporal facts concerning the earlier-than relation can obtain. Even worse, looking at reality from the general perspective is of no help whatsoever. Facts constituting reality must be obtaining somewhere. But there is no fragment in which trans-temporal fact can obtain. Therefore, the general perspective contains no trans-temporal fact. It follows that there is no way to recover an ordered sequence of fragments. But then it seems that the fragmentalist lacks the metaphysical resources to properly describe concrete particulars as spread along the temporal dimension. How can she describe stages as coming one after another, if she is simply unable to recover a temporal order in the fragmented reality? There is no way of grounding the earlier-than relation in facts connecting different fragments, for they do not exist.

My reply is the following. I am willing to admit that in the picture I'm describing no ground for facts involving the earlier-than relation can be found, at least in the canonical sense of "earlier-than relation". Still, there is a way to recover a proper temporal order. Let us see how. As I said, I treat Fragmentalist Presentism as the conjunction of Fragmentalist $\mathrm{OP}^{*}$ and Fragmentalist FP. It follows that the fragmentalist presentist is committed to exclude past and future entities from the inventory of the fragment we live in. But then, how can she evaluate as true (or false) past-tensed (or future-tensed) sentences like: "Caesar crossed the Rubicon"? The answer is simple. She cannot, unless she inflates her ontology with proxy past (and future) entities. For instance, she could opt for the Lucretian approach, by enriching the inventory of our fragment with tensed facts about the world presently instantiating properties like being such that Caesar crossed the Rubicon. These facts provide 
a ground for the truth of "Caesar crossed the Rubicon" without contradicting the presentist assumption that our fragment do not contain Caesar, not even as a past entity. Call this version of Fragmentalist Presentism, the version of Fragmentalist Presentism that is enriched with proxy past (and future) entities, Proxy Fragmentalist Presentism. This concluded the first step on my reply.

The second step is as follows. I claim that, within a proxy fragmentalist framework, fragments can be thought of as containing past (and future) facts as far as we take them to be past (and future) in a weak sense 7 What is a weak past fact? Suppose that Alan was sitting and now running. From a metaphysical point of view we can describe this case in two different ways. According to the first one, our fragment is constituted by the presenttensed fact that Alan is running and the past-tensed fact that Alan was sitting. Note that, even though the latter fact is past-tensed, it is nothing but a presently obtaining fact. This is a weak past fact. According to the second way, reality is now constituted by the present-tensed fact that Alan is running, and it was constituted by the present-tensed fact that Alan is sitting. Differently from the former fact, the latter constituted reality, which it does not now. This is a strong past fact. Strong past facts are obviously incompatible with Proxy Fragmentalist Presentism. Still, nothing prevents the fragments from containing weak past facts. The latter can be seen as nothing but tensed facts about proxy past entities. We can describe, say, the tensed facts about the world presently instantiating the property being such that Caesar crossed the Rubicon as nothing but weak past facts about Caesar's being crossing the Rubicon. This concludes the second step of my reply.

Here is the third and last step. The distinction between weak and strong facts helps us recover the coveted temporal order of fragments. Think of fragments as pieces of a puzzle. In order to put the pieces together, we are required to examine their shape. My idea is that the "shape" of a given fragment can be recovered by examining the past and future facts that obtain within it. Suppose that a fragment $F_{0}$ contains both the present-tensed fact that there are no Martian outposts and the future-tensed fact that there will be Martian outposts, while within a fragment $F_{1}$ we find both the present-tensed fact that there are Martian outposts and the past-tensed fact that there were no Martian outposts. $F_{0}$ does not contain future Martian outposts, hence within it we find only weak

\footnotetext{
7 For the distinction between weak and strong (past and) future facts see Ciuni and Torrengo (2013).
} 
future facts about them. However, nothing prevents us from finding the "corresponding" strong future facts within $F_{1}$, which contains Martian outposts as presently existing objects, while no strong past fact about the absence of Martian outposts obtains. The proxy fragmentalist presentist will now describe $F_{0}$ as temporally preceding $F_{1} \unlhd^{8}$ Notice that, even though reality is thought of as irreducibly fragmented, the order just described is rock solid: it is grounded in the "shape" of the fragments. Now we have the tools to make sense of the idea that there are perdurant objects spread out along the fragments. Suppose Alan is presently running and then sitting. Looking at the temporal dimension from the general perspective, the fragmentalist will say that there are at least two fragments, $F_{1}$ and $F_{n}$, such that $F_{1}$ precedes $F_{n}$. While the former contains the stage $A_{1}$ having the property of being running, the latter contains the stage $A_{n}$ satisfying the property of being sitting.

\section{Fragmentalist Presentist Perdurantism}

In contrast to the version of Perdurantism proposed by Brogaard, the theory of persistence just described call it Fragmentalist Presentist Perdurantism (FPP) - does justice to the idea that every stage of a given concrete particular is properly part of reality. Let me stress, once again, that looking from the general perspective reality is constituted by all the facts that obtain within each fragment. Both the present-tensed fact that $A_{1}$ is running and the present-tensed fact that $A_{n}$ is sitting constitute reality in an absolute sense. But this is only part of the story. In accordance with Brogaard's view, FPP vindicates the presentist assumption that past and future are unreal. From the current perspective, only the present is properly part of reality: there is no fragment in which the fact that $A_{1}$ is running and the fact that $A_{n}$ is sitting can obtain together. Within the fragment $F_{1}$ we find no strong fact about the existence of $A_{n}$. Similarly, within $F_{n}$ no strong fact about the existence of $A_{1}$ can obtain. $F_{1}$ will contain at most the weak future fact that $A_{n}$ will be sitting. As I stressed above, the latter is nothing but a presently obtaining fact. Analogously, $F_{n}$ will contain at most the weak past fact that $A_{1}$ was running. In other

\footnotetext{
${ }^{8}$ To keep things simple, I take the future to be linear. Those who prefer to adopt a branching time model can reformulate this argument by employing only past-tensed sentences as examples. Furthermore, I will avoid complications arising from relativistic considerations on the nature of spacetime.
} 
words, from the current perspective, as time goes by we will find the relentless succession of purely present stages described by Brogaard.

Let me now prevent another possible objection to my view. As seen in the introduction, perdurantists identify concrete particulars with mereological sums of stages from different times. But I have underlined that reality contains no trans-temporal fact: reality is not "of a whole". The fragmentalist cannot accept the existence of mereological sums of stages from different times, for the simple reason that a mereological sum whose relata belong to different times is a trans-temporal relation. It follows that, although from the general perspective every stage of Alan can be said real, no fact about the mereological sum of his stages can be grounded. In a sense, the parts are real, the whole is not. But standard perdurantists claim - as Sider (200I: 6o) has it - that "it is spacetime worms that are $[\ldots]$ the referents of our ordinary terms, members of ordinary domains of quantification, subjects of ordinary predications, and so on”. According to this view, an utterance such as 'Alan will be sitting' is true if and only if the appropriate mereological sum of stages is composed by a future stage that is sitting. Still, in light of FPP, reality cannot contain this kind of objects. So, how can we account for the truth of the utterance 'Alan will be sitting'? Adopting the stage view instead of standard perdurantism is of no help whatsoever here: similar worries arise. Stage viewers claim that the referents of the objects we usually talk about are single stages, not spacetime "worms" (Sider 1996, 2000, 200I) 9 Thus, in evaluating the truth of a present-tensed utterance they do not need to appeal to more than one stage, the present one. Still, in evaluating as true or false a pastor a future-tensed utterance, they resort to a trans-temporal relation: the relation of temporal counterparthood. In Sider (2000: 84)'s words, "a current assertion of 'Clinton was indiscreet' is true iff the (current) referent of 'Clinton' - a stage - has an indiscreet temporal counterpart in the past”. Put differently, the truth conditions for past- and future-tensed utterances offered by stage viewers presuppose the existence of facts that are clearly incompatible with the fragmentalist picture.

Here is my reply. Although the followers of FPP can resort neither to the relation of mereological sum nor to the relation of temporal counterparthood in accounting for the truth of past- and future-tensed utterances,

9 See also Hawley (200I) and Varzi (2003). 
nothing prevents them from regarding fragments as its supervenience base. Consider the utterance 'Alan will be sitting' - call it $U$. The followers of FPP will claim that the truth of the proposition $U$ expresses in a given fragment modally co-varies with the facts obtaining in the other fragments. If $U$ expresses a true proposition $P$ in a fragment $F_{1}$, even though within $F_{1}$ we will find nothing but the (presently obtaining) future-tensed fact that $A_{n}$ will be sitting, there will be another fragment, $F_{n}$, such that it contains the present-tensed fact that $A_{n}$ is sitting, that is, the fact which $P$ corresponds to. Conversely, if $P$ were false we would find neither the future-tensed fact that $A_{1}$ will be sitting obtaining in $F_{1}$ nor the present-tensed fact that $A_{n}$ is sitting obtaining in $F_{n}$. This modal co-variation, which is clearly a supervenience relation between the truth of the propositions expressed by past- and future-tensed utterances and the content of each fragment, is all what the followers of FPP need in order to account for past- and future-tensed truths.

A similar reply can be offered - I think - to a more sophisticated objection, made by Tallant (2018) ${ }^{\text {I0 }} \mathrm{He}$ claims that perdurantists and stage viewers are bound to accept transtemporal identity dependencies and that these dependencies are incompatible with the presentist ontologym Following Lowe (2010), he characterises the notion of identity dependence as follows.

$$
\begin{aligned}
& \text { Identity Dependence } \quad x \text { depends for its identity upon } y={ }_{d f} \text { There is a function } f \text { such that it is part of the essence of } \\
& \quad x \text { that } x \text { is } f(y) .
\end{aligned}
$$

As an example, Lowe considers a marriage $x$ between two people $y$ and $z$. Since "the identity of a marriage depends on the identities of the two people being married" (20IO: $\S 4$ ), saying that $x$ depends for its identity upon $y$ will be tantamount to saying that there is a function $f$, that is, the marriage with $z$ function, such that it is part of the essence of $x$ that $x$ is the $f$ of $y$. Similar considerations can be extended to mereological sums. A

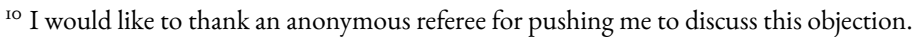

II Tallant's arguments are not limited to perdurantism and stage view, since he holds that even the conjunction of presentism and endurantism proves to be problematic. Discussing the relations between presentism and endurantist theories of persistence would be far beyond the scope of this paper. For this reason, I will set aside an argument that Tallant proposes in order to attack "the general idea that presentism is compatible with identity over time" (2018: 2213), focusing on the arguments that explicitly involve perdurantism and the stage view.
} 
sum $x$ - Tallant argues $\sqrt{12}$ - depends for its identity upon its parts $y$. This means that there is a function $f$, which Tallant calls the "having $y$ as a part" function, such that it is part of the essence of $x$ that $x$ is the $f$ of $y$ (in other words, it is part of the essence of $x$ that $y$ is a part of $x$ ). Now suppose that $x$ stands for a mereological sum of stages from different times. The trouble for the presentist is that, since she takes no more than one stage at a time to be real, she can at best say that $x$ depends for its identity upon its present parts. But given that which stages are present changes as time goes by, the identity of the sum itself will change as time goes by (Tallant 2018: 2212). The unwelcome conclusion is that no object can persist.

This argument can also be applied to the stage view. Reconsider Alan. In Tallant's words:

Qua persisting object, [Alan] depends for his existence upon the existence of stages that exist at times other than the present. The stages would not be identical to [Alan], unless they were counterpart related to [Alan]. Thus, these other stages depend for their identity on being counterpart theoretic related to [Alan]. This seems a clear case of identity dependence (Tallant 2018: 2213. Italics in the original).

In this case, saying that a stage $x$ of Alan depends for its identity upon Alan is tantamount to saying that there is a function $f$, that Tallant calls the "being counterpart theoretic related to [Alan]" function, such that it is part of the essence of $x$ that $x$ is the $f$ of $y$ (it is part of the essence of $x$ that $x$ is counterpart theoretic related to Alan). Once again, the presentist has a problem here, since Alan is the only stage that can be real. But then there is no past or future stage than can be counterpart theoretic related to Alan (Tallant 20I8: 22I3). The absurd conclusion follows that Alan does not persist.

My reply is as follows. I am happy to concede that Tallant's arguments succeed in showing that presentism and perdurantism (or the stage view) are jointly incompatible, but only if we limit our attention to standard presentism. The reason why I opt for a non-stardard version of presentism is precisely that I take the standard one to be incompatible with perdurantism (and the stage view). And, as I stressed above, I also concede that,

I2 See also Lowe (2012: 95). 
in order to combine my version of fragmentalism with perdurantism, we need to somehow modify our understanding of the latter: my fragmentalist cannot accept the existence of mereological sums of stages from different times, for she rejects the claim that reality contains trans-temporal facts (reality is not "of a whole"; for the same reason, she rejects the notion of temporal counterparthood). Still, in contrast to the standard presentist, she has the ontological resources to vindicate the idea that every stage of a concrete particular is real. Looking from the general perspective, all the fragments are on a par, in the sense that, from the general perspective, reality is constituted by facts involving all the stages of a concrete particular. And in section 3 I showed that the rejection of trans-temporal facts, including facts about the existence of mereological sums of stages from different times, does not prevent the fragmentalist from recovering an ordered sequence of fragments. This, in turn, allows her to recover an ordered sequence of stages. This is the reason why she can ultimately describe concrete particulars (or counterpart theoretic related stages) as spread throughout the temporal dimension.

\section{References}

Benovsky, J. 2009. Presentism and Persistence. Pacific Philosophical Quarterly 90: 29I-309

Brogaard, B. 200o. Presentist Four-Dimensionalism. The Monist 83: 34I-354

Ciuni, R., Torrengo, G. 20I3. Presentism and Cross-Temporal Relations. In R. Ciuni, K. Miller, G. Torrengo (Eds.). New Papers on the Present. Focus on Presentism. Munich: Philosophia Verlag

Fine, K. 2005. Tense and Reality. In K. Fine. Modality and Tense. Oxford: OUP, pp. 26I-320

Fine, K. 2006. The Reality of Tense. Synthese 150: 399-414 
Hawley, K. 20oI. How Things Persist. Oxford: Clarendon Press

Heller, M. 1992. Things Change. Philosophy and Phenomenological Research 52: 695-704

Lewis, D. 1986. On the Plurality of Worlds. Oxford: Blackwell

Lipman, M. 2015. On Fine's Fragmentalism. Philosophical Studies 172: 3119-3133

Lombard, L. 1986. Events: A Metaphysical Study. London: Routledge and Kegan Paul

Lowe, E.J. 2oIo. Ontological Dependence. In E.N.Zalta (Ed.). The Standford Encyclopedia of Philosophy (Spring 20Io Edition).http://plato.stanford.edu/archives/spr2010/entries/dependence-ontological/

Lowe, E.J. 20I2. Against Monism. In P. Goff(Ed.). Spinoza on Monism. London: Palgave Macmillan, pp. 92-I22

McTaggart, J.M.E. 1908. The Unreality of Time. Mind I7: 457-474

Sider, T. 1996. All the World's a Stage. Australasian Journal of Philosophy 74: 433-453

Sider, T. 2000. The Stage View and Temporary Intrinsics. Analysis 6o: 84-88

Sider, T. 20or. Four-Dimensionalism. An Ontology of Persistence and Time. Oxford: OUP

Tallant, J. 20I8. Presentism, Persistence and Trans-temporal Dependence. Philosophical Studies I75: 2209-2220 
Varzi, A. 2003. Naming the Stages. Dialectica 57: 387-412 